\title{
Morbimortalidade por COVID-19 associada a condições crônicas, serviços de saúde e iniquidades: evidências de sindemia
}

\author{
Tatiana Pestana Barbosa, ${ }^{1}$ Fernanda Bruzadelli Paulino da Costa, ${ }^{1}$ Antônio Carlos Vieira Ramos, ${ }^{1}$ \\ Thaís Zamboni Berra, ${ }^{1}$ Luíz Henrique Arroyo, ${ }^{1}$ Yan Mathias Alves, ${ }^{1}$ Felipe Lima dos Santos ${ }^{1}$ \\ e Ricardo Alexandre Arcêncio ${ }^{1}$
}

Como citar

Barbosa TP, Costa FBP, Ramos ACV, Berra TZ, Arroyo LH, Alves YA, et al. Morbimortalidade por COVID-19 associada a condições crônicas, serviços de saúde e iniquidades: evidências de sindemia. Rev Panam Salud Publica. 2022;46:e6. https://doi.org/10.26633/RPSP.2022.6

RESUMO

Objetivo. Identificar os fatores correlacionados à incidência e mortalidade por COVID-19 e verificar situações de sindemia em escala global.

Métodos. Realizou-se um estudo ecológico de casos e óbitos confirmados de COVID-19 a partir de informações coletadas do European Center for Disease Prevention and Control em 2019 e 2020. Para a caracterização dos países, utilizaram-se indicadores do Banco Mundial e Worldometer Coronavirus. Foram realizadas análises descritivas e de correlação entre as variáveis independentes para posteriormente realizar o modelo de regressão linear múltipla, com o objetivo de identificar os fatores correlacionados à incidência e mortalidade por COVID-19.

Resultados. Obtiveram-se dados de 185 países. A média da incidência dos casos foi de 16 482/mil habitantes, enquanto a média para mortalidade por COVID-19 foi de 291/mil habitantes, sendo América do Norte e Leste Asiático e Pacífico as regiões que apresentaram maiores e menores índices, respectivamente. Identificouse correlação positiva da taxa de incidência com proporção da população com idade de 15 a 64 anos, população urbana, desigualdade conforme Índice de Gini e com seis das sete regiões analisadas (exceto Leste Asiático e Pacífico). A taxa de mortalidade apresentou correlação negativa com a população de 0 a 14 anos e positiva com população urbana, desigualdade conforme índice de Gini e todas as regiões analisadas, exceto Leste Asiático e Pacífico.

Conclusões. A morbimortalidade da COVID-19 esteve correlacionada à carga de condições crônicas, ao envelhecimento da população e à baixa capacidade dos serviços de saúde para testagem e oferta de leitos hospitalares, quadro agravado em países ou regiões com elevada desigualdade social, caracterizando uma situação de sindemia.

Palavras-chave COVID-19; atenção à saúde (saúde pública); sindemia; pandemias; epidemiologia.

Desde o primeiro relato da síndrome respiratória aguda grave causada por coronavírus 2 (SARS-CoV-2), mais de 200 milhões de pessoas foram infectadas e mais de 4 milhões de pessoas morreram em todo o mundo, conforme dados de agosto de 2021 (1). Como resposta, as recomendações imediatas da Organização Mundial da Saúde (OMS) visaram à rápida identificação, ao diagnóstico e manejo dos casos e à implementação de um sistema de isolamento e rastreamento de contatos (1).

\footnotetext{
1 Escola de Enfermagem de Ribeirão Preto da Universidade de São Paulo (EERPUSP), Programa de Pós-Graduação Enfermagem em Saúde Pública, Ribeirão Preto (SP), Brasil. $\bowtie$ Tatiana Pestana Barboa, tatipestana@usp.br
} 
Há fortes evidências de que pessoas com condições crônicas e idosos são mais vulneráveis às manifestações graves e à mortalidade pela COVID-19 (2). Além disso, a doença teve impactos na estrutura e organização dos sistemas e serviços de saúde, uma vez que houve necessidade de investimentos e reorganização desses serviços para minimizar os prejuízos no atendimento de outras demandas de saúde (2). Soma-se a isso a observação de que as regiões com maiores índices de desigualdade social tiveram pior desempenho no enfrentamento da COVID-19, apresentando incidência e mortalidade mais acentuadas (3).

Dessa maneira, ressalta-se que nem todos os contextos serão igualmente afetados pela COVID-19, tendo em vista que a influência do vírus não será unilateral, mas sim mediada pela interação com outras doenças, principalmente as doenças crônicas não transmissíveis (DCNT). A interação entre condições preexistentes e os clusters de casos de COVID-19, impulsionados ainda por fatores políticos, econômicos e sociais, caracteriza a situação como uma sindemia (4). Compreender a COVID-19 como uma sindemia fornece estratégias poderosas para entender como os fatores sociais, políticos e ecológicos criam e perpetuam vulnerabilidades estruturais que contribuem para o surgimento e a exacerbação de doenças e agravos à saúde (5).

Tendo-se que as iniquidades sociais mudam de acordo com o contexto no qual o indivíduo está inserido, podendo afetar diretamente sua compreensão acerca da doença e dos meios de prevenção, ressalta-se a importância dos estudos ecológicos que conseguem avaliar como os contextos social e ambiental podem afetar determinados grupos populacionais. Assim, o presente estudo teve como objetivo identificar os fatores correlacionados à incidência e à mortalidade por COVID-19 e verificar situações de sindemia em escala global, considerando dados demográficos, carga de DCNT, capacidade dos serviços de saúde em oferecer testes diagnósticos, número de leitos hospitalares e desigualdade social.

\section{MATERIAIS E MÉTODOS}

Desenvolveu-se um estudo ecológico (6), em escala global, que utilizou fontes de dados secundárias. Para o estudo, foi considerada a subdivisão de regiões do mundo proposta pelo Banco Mundial: 1) Leste Asiático e Pacífico; 2) Europa e Ásia Central; 3) América Latina e Caribe; 4) Oriente Médio e Norte da África; 5) América do Norte; 6) Sul da Ásia; e 7) África Subsaariana.

Os dados referentes à COVID-19 foram coletados do European Center for Disease Prevention and Control (ECDC), que disponibiliza informações atualizadas diariamente sobre a doença por meio de uma busca ampla em diferentes fontes de dados mundiais, como websites de ministérios e de instituições de saúde pública dos países (7). O número de habitantes de cada país foi obtido do Banco Mundial (8). A coleta dessas duas fontes foi realizada no dia 8 de janeiro de 2021 .

Foram analisados os casos e óbitos diagnosticados e confirmados de COVID-19 que ocorreram de 31 de dezembro de 2019 a 31 de dezembro de 2020. Destaca-se que foram utilizados dados da população geral. As taxas de incidência e mortalidade foram calculadas a partir do número de casos e óbitos dividido pela população de cada país e multiplicado por 100000 como constante.
Para a caracterização dos países, foram utilizados indicadores disponíveis no World Development Indicators do Banco Mundial (8): proporção da população com idade $\leq 14$ anos; proporção da população com idade de 15 a 64 anos; proporção da população com idade $\geq 65$ anos; prevalência de diabetes ( $\%$ da população de 20 a 79 anos de idade); causa da morte por DCNT (\% do total de óbitos no país); leitos hospitalares (por 1000 habitantes); população urbana (\% da população total); e desigualdade social, expressa pelo Índice de Gini, uma medida para mensurar como a renda de um país é distribuída entre a população, sendo considerado um indicador de desigualdade social. Seus valores podem variar de 0 a 100\% (ou 0 a 1, a depender da apresentação). Quanto maior for o valor do índice de Gini, maior é a desigualdade na distribuição da renda entre a população no país; quanto mais próximo de zero for o valor, maior é a igualdade na distribuição de renda.

Foram utilizados dados da população geral (residentes em áreas urbanas e rurais) para os cálculos das taxas de incidência e mortalidade por COVID-19. A porcentagem de população urbana foi utilizada como variável independente, pois, segundo a literatura, as doenças infecciosas se originam ou se propagam mais rapidamente nas cidades em comparação às áreas rurais por causa da urbanização. Vale destacar que, segundo a definição do Banco Mundial, a variável "causa da morte por DCNT" refere-se às doenças crônicas não transmissíveis e inclui câncer, diabetes, anomalias congênitas e doenças cardiovasculares, digestivas, de pele e musculoesqueléticas, sendo realizado o cálculo da proporção de todas as mortes por essas condições como causa básica, considerando todas as idades (8).

O número de testes realizados (por 1 milhão de habitantes) para o diagnóstico da COVID-19 em cada região/país foi obtido no Worldometer Coronavirus (https: / /www.worldometers.info/ coronavirus/), coletado no dia 8 de janeiro de 2021 (9). Como o Worldometer utiliza as estatísticas de testes fornecidas pelos órgãos oficiais dos países, não é possível identificar e descrever quais testes (ou critérios) foram realizados para o diagnóstico da COVID-19.

Para analisar os fatores correlacionados à incidência e mortalidade de COVID-19 e, assim, identificar situações de sindemia, recorreu-se à regressão linear múltipla (10). Optou-se pelo desenvolvimento de duas modelagens de forma independente: uma considerando a incidência e a outra, a mortalidade como variável dependente. As variáveis independentes consideradas em ambos os modelos foram aquelas coletadas do World Development Indicators e Worldometer Coronavirus e citadas anteriormente.

Com o intuito de minimizar potenciais fatores de confusão, foram identificados e explorados os valores ausentes de todas as variáveis nos estágios iniciais da análise, utilizando o pacote "naniar" do software R, que fornece estruturas de dados e funções que facilitam a plotagem de valores ausentes. Isso permitiu que dependências de dados ausentes fossem exploradas com desvio mínimo dos padrões de trabalho (11). Após a etapa de verificação dos valores ausentes, foi realizada análise exploratória do novo banco de dados, seguida da análise descritiva das variáveis, dos gráficos de dispersão e dos boxplots.

Foram realizadas análises de correlação com as variáveis independentes numéricas a fim de conhecer os candidatos a serem inseridos no modelo de regressão linear múltipla. Nessa etapa, foi utilizada a função loess (10), que ajusta uma superfície polinomial determinada por um ou mais preditores numéricos, usando ajuste local. 
Para reduzir a assimetria nas taxas de incidência e mortalidade, foi feita uma transformação logarítmica (log) que pertence à família mais geral de transformações Box-Cox (10). Para um número real $\lambda \neq 0$, uma transformação Box-Cox T $\lambda$ é definida como $\mathrm{T} \lambda(\mathrm{x})=(\mathrm{x} \lambda-1) / \lambda$, em que $\mathrm{x}$ é uma variável positiva. No caso limite $\lambda=0$, temos $\lim \lambda \rightarrow 0(x \lambda-1) / \lambda \rightarrow \log (x)$; então podemos definir $\mathrm{T} 0(\mathrm{x})=\log (\mathrm{x})$ e considerar a transformação de $\log$ como um caso especial de transformações Box-Cox (12). Foi necessário obter o valor do parâmetro $\lambda$ para encontrar os valores dos dados transformados e utilizar esses dados transformados para efetuar as análises, sendo utilizado o pacote MASS (13).

Para construir o modelo de regressão, utilizou-se a função ols_step_backward_aic do pacote olsrr (14), que remove cada preditor com base nos valores do critério de informação de Akaike (Akaike information criterion, AIC), de maneira gradual, até não haver mais variáveis para remover. Após obtenção do modelo final para cada variável dependente (incidência e mortalidade por COVID-19), foram realizados testes para verificar a normalidade dos resíduos, com o gráfico QQ Plot e histogramas (15).

Para verificar a heterocedasticidade do modelo, recorreu-se ao teste multiplicador de Lagrange (16) para detectar a dependência transversal do modelo. A validade assintótica do procedimento do teste é baseada no número de observações de séries temporais $\mathrm{T}$, sendo grande em relação ao número de unidades de seção transversal N, que, por sua vez, é baseado na média dos coeficientes de correlação da amostra de pares quadrados dos resíduos e é aplicável quando $\mathrm{N}$ é fixo e $\mathrm{T} \rightarrow \infty$; ou seja, quando N é pequeno em relação a um T grande (17).

Ainda, para verificar a heterocedasticidade, foi utilizada a função ols_test_breusch_pagan do pacote olsrr, que se baseia no qui-quadrado e no valor de $P$; para valores $P<0,05$, rejeita-se a hipótese nula de variância constante.

Além disso, a multicolinearidade das variáveis independentes foi verificada através do valor de VIF (variance inflation factor). Fatores com VIF > 10 apresentam problemas de multicolinearidade; portanto, as variáveis que obtiveram esse valor foram removidas do modelo (14). Para verificação, aplicou-se a função ols_coll_diag do pacote olsrr.

Recorreu-se também ao AIC para escolher o melhor modelo para análise do material empírico. O AIC estima a probabilidade de o modelo prever valores futuros, que é penalizada pelo número de parâmetros estimados no modelo. Ao automatizar o processo de calibração do modelo, o mesmo pode ser testado com todas as combinações possíveis de variáveis de entrada. $\mathrm{O}$ melhor padrão é identificado pela seleção da combinação de variáveis que resulta no menor valor de AIC, considerado o modelo ótimo para previsão $(18,19)$. Por fim, para verificar a normalidade dos resíduos, realizou-se o teste de Kolmogorov-Smirnov.

Devido ao uso de dados secundários de domínio público, o estudo teve dispensa de aprovação de um Comitê de Ética em Pesquisa. As análises deste estudo foram realizadas usando o software R versão 4.0.1.

\section{RESULTADOS}

Foram obtidos dados de 185 países que possuíam um bom percentual de preenchimento dos nove indicadores demográficos, socioeconômicos e de saúde analisados. A taxa de incidência da COVID-19 nos países variou de 3 a 79068 casos por 100000 habitantes, com mediana de 7886 e média de 16482 casos por 100000 habitantes. Quanto à taxa de mortalidade por COVID-19, houve variação de zero (países sem óbitos registrados) a 1724 óbitos para cada 100000 habitantes, com mediana de 86 e média de 291 casos por 100000 habitantes.

A distribuição das taxas de incidência e mortalidade por COVID-19 segundo regiões do mundo pode ser observada nas figuras 1 e 2 , respectivamente. As regiões que apresentaram as maiores medianas de taxas de incidência e mortalidade da doença (casos/100 mil habitantes) foram América do Norte, Europa e Ásia Central; enquanto que os menores índices foram observados na América Latina, Caribe e Sul da Ásia para a incidência e no Sul da Ásia e África Subsaariana para a mortalidade.

Quanto às variáveis selecionadas para caracterização dos países, as regiões que apresentaram maiores e menores medianas, respectivamente, foram: Oriente Médio e Norte da África e Sul da Ásia para número de leitos hospitalares; Oriente Médio e Norte da África e África Subsaariana para prevalência de diabetes; Europa e Ásia Central e África Subsaariana para causa da morte por DCNT; Oriente Médio e Norte da África e Europa e Ásia Central para proporção da população com idade $\leq 14$ anos; Oriente Médio e Norte da África e África Subsaariana para proporção da população com idade de 15 a 64 anos; América do Norte e Sul da Ásia para proporção da população com idade $\geq 65$ anos; América Latina e Caribe e Europa e Ásia Central para Índice de Gini; América Latina e Caribe e África Subsaariana para testes para COVID-19 realizados (tabela 1). Com o objetivo de conhecer as variáveis independentes candidatas ao modelo, realizou-se análise de correlação, evidenciando correlação positiva entre a incidência e as variáveis mortes por DCNT, população com mais de 65 anos e número de testes realizados; foi identificada correlação negativa com a população de 0 a 14 anos.

Quanto à mortalidade, os resultados evidenciaram correlação positiva com mortes por condições crônicas e população com mais de 65 anos, além de correlação negativa com população com idade de 0 a 14 anos. Para confirmar a suposição de não correlação entre as variáveis dependentes (taxas de incidência e mortalidade) com as variáveis de leitos hospitalares, prevalência de diabetes, proporção total da população com idade de 15 a 64 anos e número de testes realizados na população, foi testado o efeito quadrático no modelo final, não sendo observada mudança estatística significativa. Foi realizada transformação Box-Cox devido ao fato de a distribuição das taxas de incidência e mortalidade se encontrarem enviesadas, sendo utilizada estimativa de máxima verossimilhança. Dessa maneira, foi encontrado valor de lambda próximo a 0,20 para a incidência e lambda próximo a 0,15 para a mortalidade, indicando uma transformação logarítmica no modelo.

Na tabela 2, é possível verificar os resultados obtidos e as variáveis correlacionadas às taxas de incidência e mortalidade por COVID-19, respectivamente. Referente à taxa de incidência, foi possível identificar correlação positiva com as variáveis estatisticamente significativas $(P<0,05)$ proporção da população de 15 a 64 anos, população urbana e desigualdade conforme Índice de Gini (quanto maior a desigualdade social, maior o índice de Gini e maior a taxa de incidência de COVID-19); além da correlação também positiva com todas as regiões analisadas, exceto com a Região 1 (Leste Asiático e Pacifico), que não entrou no modelo final. 
FIGURA 1. Distribuição das taxas de incidência de COVID-19 por regiões do mundo, segundo subdivisão do Banco Mundial

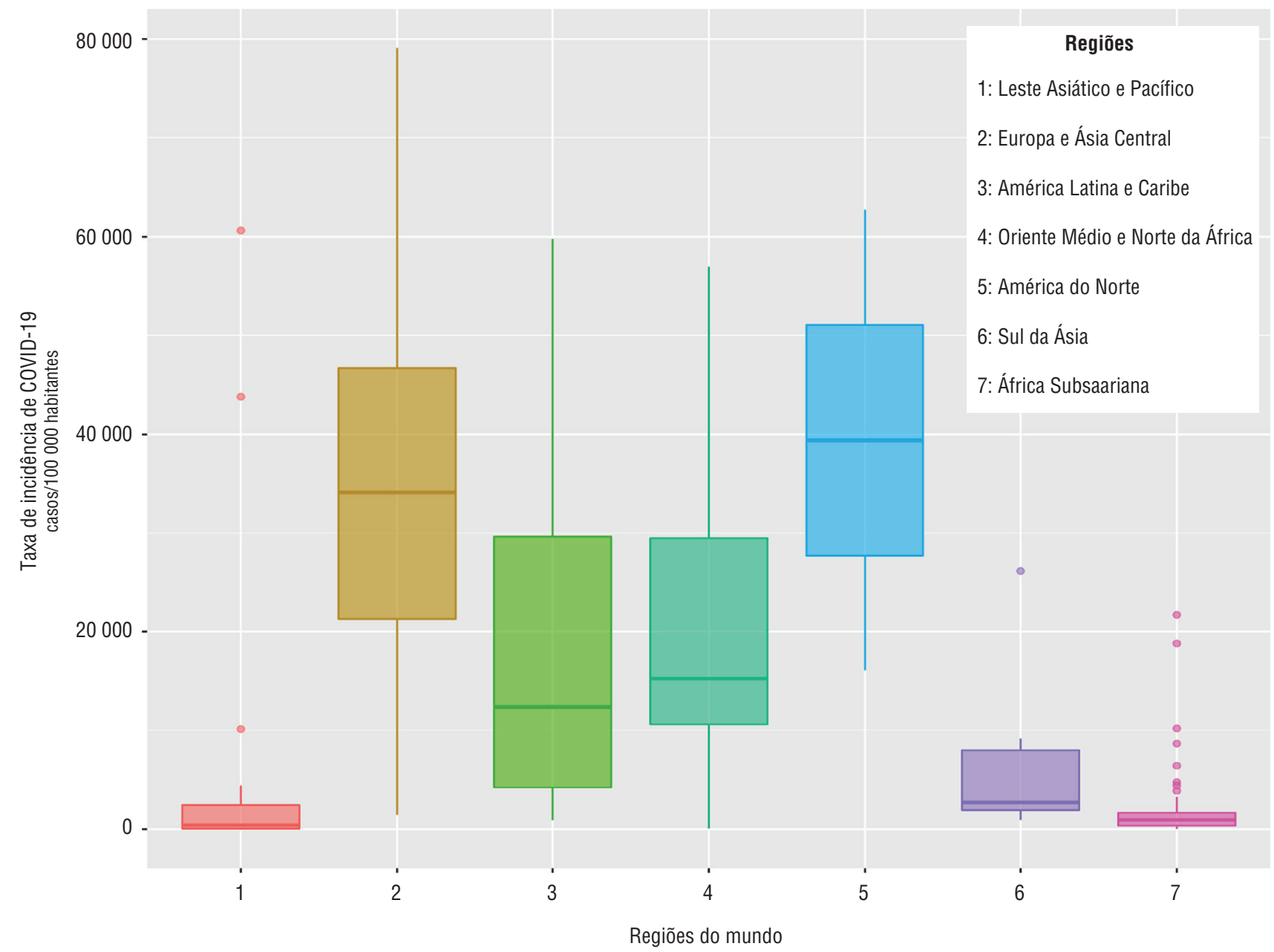

Fonte: European Center for Disease Prevention and Control (dezembro/2019 a dezembro/2020).

Referente à mortalidade por COVID-19, identificou-se correlação negativa com a variável população de 0 a 14 anos e positiva com as variáveis população urbana e desigualdade social, essa última expressa pelo índice de Gini, além da correlação positiva com todas as regiões analisadas, exceto com a Região 1 (Leste Asiático e Pacífico), que não entrou no modelo final. Destaca-se que foi verificada variância constante e normalidade dos resíduos apresentados através dos gráficos Residuals vs. Fitted, QQ-Plot e histograma dos resíduos. O material está disponível para consulta mediante solicitação. Pela aplicação do teste KolmogorovSmirnov, observou-se o valor de $P=0,35$ para a incidência transformada e de $P=0,26$ para a mortalidade, não sendo, portanto, rejeitada a hipótese nula de normalidade dos resíduos.

\section{DISCUSSÃO}

O presente estudo buscou identificar os fatores correlacionados à incidência e mortalidade por COVID-19 e verificar situações de sindemia em escala global. $\mathrm{O}$ estudo evidenciou que o envelhecimento, uma capacidade limítrofe dos serviços de saúde de oferecer testagem em massa, a cobertura de leitos hospitalares e contextos de grandes desigualdades sociais estiveram correlacionados não somente à disseminação da COVID-19, traduzida pela incidência, como também à gravidade dos casos, que culminaram em muitas mortes. Esse quadro demonstra uma interação entre a COVID-19 e diversos aspectos contextuais (sociais, epidemiológicos e dos sistemas de saúde), caracterizando uma situação de sindemia. Vale destacar que existem diversos indicadores de serviços de saúde; porém, optou-se por utilizar o número de leitos hospitalares, visto que os casos mais graves, em sua maioria correlacionados às condições crônicas, eram bastante complexos. Portanto, a disponibilidade de leitos tem sido crucial para a recuperação dessas pessoas e melhores chances de sobrevida.

A América do Norte foi a região que apresentou as maiores taxas de incidência e mortalidade por COVID-19, segundo o recorte temporal do estudo. Essa região apresentou o maior número de testes realizados no período investigado, bem como a maior proporção de mortes por condições crônicas, indicando que possui uma população mais suscetível à gravidade da infecção por COVID-19 (19). Destaca-se que essa região apresenta uma grande concentração de metrópoles (especialmente nos Estados Unidos e Canadá), evidenciando que grandes centros 
FIGURA 2. Distribuição das taxas de mortalidade por COVID-19 por regiões do mundo, segundo subdivisão do Banco Mundial

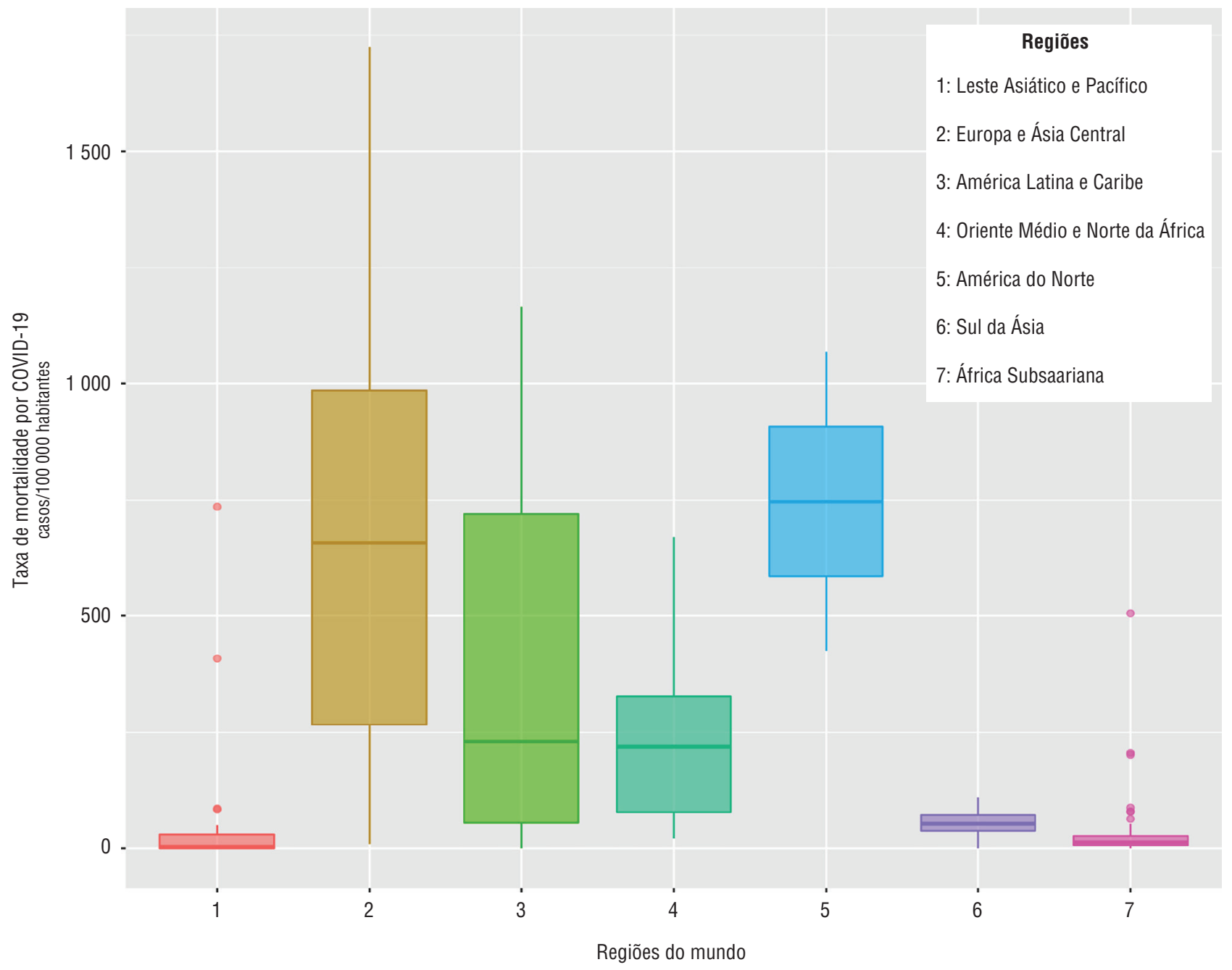

Fonte: European Center for Disease Prevention and Control (dezembro/2019 a dezembro/2020).

urbanos são importantes locais para a circulação e disseminação da COVID-19 (20, 21).

Os fatores de risco para mortalidade por COVID-19, principalmente as condições crônicas, são cada vez mais comuns em países de baixa e média renda, especialmente entre indivíduos com níveis educacionais mais baixos (22). Em relação à desigualdade social, observou-se uma correlação positiva com as taxas de incidência e mortalidade por COVID-19, evidenciando o caráter social da pandemia.

Historicamente, a Região da América Latina e Caribe está entre as que apresentam os maiores índices de desigualdades sociais, com índice de Gini médio de 46,01 (23), refletindo um cenário no qual as iniquidades sociais estão bastante presentes. Portanto, o baixo número de testes realizados nessa região pode influenciar a subnotificação e o subdiagnóstico de casos e óbitos, levando à existência de uma prevalência oculta da doença no continente. O substancial crescimento da COVID-19 observado na Região das Américas foi, e ainda é, preocupante, uma vez que o centro e o sul do continente compreendem principalmente países em desenvolvimento, cujas populações dependem de sistemas públicos de saúde, que sofreram uma sobrecarga com o aumento descontrolado da transmissão da doença e consequente aumento das necessidades assistenciais nesses locais (23).

É inviável pensar em um plano único para o enfrentamento da pandemia da COVID-19, visto que há um padrão social e econômico muito distinto entre os diversos países. Notadamente nos países em desenvolvimento, há quadros elevados de desigualdade social, em que grande parte da população carece de bens essenciais e serviços (23). $\mathrm{O}$ acesso inadequado a saúde, alimentação e moradia e aspectos relacionados a insegurança financeira e discriminação, entre outros, também podem impactar significativamente o número de casos e o desfecho nas populações vulneráveis desses países. Portanto, há necessidade urgente de reduzir tais vulnerabilidades e desigualdades sociais para que o enfrentamento da pandemia em todo o mundo seja mais efetivo (24). 
TABELA 1. Análise descritiva das variáveis independentes por regiões do mundo, segundo subdivisão do Banco Mundial

\begin{tabular}{|c|c|c|c|c|c|c|c|}
\hline & \multicolumn{7}{|c|}{ Região $0^{\mathrm{a}}$} \\
\hline & 1 & 2 & 3 & 4 & 5 & 6 & 7 \\
\hline \multicolumn{8}{|c|}{ Número de leitos (por 1000 habitantes) } \\
\hline Média & 3,58 & 05,07 & 2,69 & 1,96 & 2,80 & 1,56 & 1,30 \\
\hline Mediana & 2,40 & 4,70 & 1,60 & 1,60 & 2,80 & 0,75 & 0,90 \\
\hline \multicolumn{8}{|c|}{ Prevalência de diabetes (\% da população de 20-79 anos) } \\
\hline Média & 10,29 & 6,40 & 9,99 & 10,90 & 9,20 & 10,76 & 5,44 \\
\hline Mediana & 6,95 & 6,10 & 10,00 & 10,10 & 9,20 & 9,75 & 4,50 \\
\hline \multicolumn{8}{|c|}{ \% de mortes por doenças crônicas não transmissíveis } \\
\hline Média & 74,05 & 89,83 & 75,13 & 73,65 & 88,00 & 66,75 & 38,23 \\
\hline Mediana & 74,00 & 90,00 & 75,00 & 76,50 & 88,00 & 66,50 & 35,00 \\
\hline \multicolumn{8}{|c|}{ Proporção (\%) da população com idade de 0-14 anos } \\
\hline Média & 25,50 & 17,88 & 24,49 & 26,88 & 17,29 & 29,21 & 40,67 \\
\hline Mediana & 24,23 & 16,15 & 23,75 & 27,21 & 17,29 & 27,38 & 41,85 \\
\hline \multicolumn{8}{|c|}{ Proporção (\%) da população com idade de $15-64$ anos } \\
\hline Média & 66,01 & 65,91 & 66,00 & 67,78 & 66,19 & 65,28 & 56,00 \\
\hline Mediana & 65,60 & 65,40 & 65,34 & 65,90 & 66,19 & 65,28 & 56,00 \\
\hline \multicolumn{8}{|c|}{ Proporção (\%) da população com idade $\geq 65$ anos } \\
\hline Média & 8,49 & 16,21 & 9,51 & 5,34 & 16,52 & 5,52 & 3,34 \\
\hline Mediana & 6,27 & 18,34 & 8,48 & 4,39 & 16,52 & 5,45 & 2,94 \\
\hline \multicolumn{8}{|c|}{ População urbana (\% da população total) } \\
\hline Média & 57,54 & 68,05 & 64,84 & 77,50 & 81,84 & 31,47 & 43,15 \\
\hline Mediana & 57,70 & 68,14 & 67,71 & 80,10 & 81,84 & 35,33 & 42,42 \\
\hline \multicolumn{8}{|c|}{ Índice de Ginip ${ }^{b}$} \\
\hline Média & 36,22 & 31,85 & 46,34 & 34,38 & 37,60 & 35,00 & 43,98 \\
\hline Mediana & 36,40 & 32,30 & 45,50 & 33,70 & 37,60 & 33,50 & 42,42 \\
\hline \multicolumn{8}{|c|}{ No. de testes realizados (por milhão de habitantes) } \\
\hline Média & 109635,7 & 414090,5 & 119628,3 & 420358,5 & 568361,5 & 152095,1 & 39390,85 \\
\hline Mediana & 304510,0 & 332468,0 & 851530,0 & 173462,0 & 568361,5 & 482250,0 & 142645,0 \\
\hline
\end{tabular}

Em algumas áreas, o distanciamento social pode significar manter-se em áreas de favelas e/ou aglomerados subnormais, com grande densidade populacional e concentração de pessoas. Em algumas regiões, a pobreza e a desigualdade social são prevalentes, assim como as doenças tidas como negligenciadas e as DCNT. Quando co-ocorrem com a COVID-19, pode haver uma verdadeira catástrofe e crise humanitária (23). Portanto, as sindemias explicitam como os fatores políticos e sociais impulsionam, perpetuam e agravam o surgimento e o acúmulo de doenças. Diante desse contexto, destaca-se a importância das ações sociais e sanitárias para combater o avanço do vírus em escala mundial, utilizando estratégias comunitárias e tecnologias em saúde para o acompanhamento da disseminação da doença e do número de casos infectados e mortos. Tais ações podem ser executadas com base nas evidências científicas sobre os padrões da doença ao redor do mundo, pois contribuem para a caracterização dos locais mais vulneráveis (25).

O presente estudo apresenta limitações, como o fato de terem sido utilizadas bases de dados secundárias, com possíveis inconsistências na qualidade e quantidade das informações, bem como uma possível presença de dados ignorados ou incompletos. De fato, a disponibilidade limitada de dados referentes à saúde da população é um dos principais desafios na avaliação da situação de saúde nos países em desenvolvimento, visto que faltam dados de vigilância para os principais agravos de importância para a saúde pública global. Vale destacar que os métodos de diagnóstico, notificação e monitoração dos agravos diferem entre os países (até mesmo regiões dentro de um país em específico) em termos de capacidade e disposição para coletar ou informatizar essas informações. A fim de melhorar a confiabilidade e comparabilidade internacional, a OMS elabora estimativas de acordo com modelos epidemiológicos e padrões estatísticos (26). Ainda, este estudo não se aprofundou nos diferentes tipos de medidas de contenção que os países implementaram, que podem ter influenciado a magnitude da doença nesses locais e no mundo. Portanto, verifica-se a importância de realizar novos estudos com diferentes abordagens metodológicas e analíticas para compreender a situação epidemiológica da COVID-19.

O modelo focalizado na doença e no tratamento, em detrimento da prevenção, é um dos principais responsáveis pelo quadro de morbimortalidade da COVID-19, vigorando com força principalmente em contextos de grandes desigualdades. Os gestores locais devem estar atentos aos riscos e às consequências desse modelo, sob pena de novas catástrofes. Elaborar políticas públicas orientadas pelas condições crônicas e pelos 
TABELA 2. Modelo de regressão linear para as taxas mundiais de incidência e mortalidade por COVID-19 (dezembro/2019 a dezembro/2020)

\begin{tabular}{|c|c|c|c|c|}
\hline \multirow[b]{2}{*}{ Variáveis independentes } & \multicolumn{2}{|c|}{ Taxa de incidência } & \multicolumn{2}{|c|}{ Taxa de mortalidade } \\
\hline & $\mathrm{IC95 \%}$ & Valor de $P$ & IC95\% & Valor de $P$ \\
\hline Intercepto ${ }^{\mathrm{a}}$ & $-5,38 ;-0,70$ & 0,130 & $-1,36 ;-2,80$ & 0,496 \\
\hline \multicolumn{5}{|l|}{ Regiãob } \\
\hline 2 & 3,$67 ; 4,99$ & $<0,001^{c}$ & 3,$23 ; 4,73$ & $<0,001^{c}$ \\
\hline 3 & 2,$29 ; 3,89$ & $<0,001^{c}$ & 2,$20 ; 3,94$ & $<0,001^{c}$ \\
\hline 4 & 2,$41 ; 4,13$ & $<0,001^{\mathrm{C}}$ & 2,$27 ; 4,16$ & $<0,001^{\mathrm{C}}$ \\
\hline 5 & 2,$28 ; 5,50$ & $<0,001^{\mathrm{c}}$ & 2,$05 ; 5,58$ & $<0,001^{c}$ \\
\hline 6 & 2,$48 ; 4,46$ & $<0,001^{c}$ & 0,$87 ; 3,03$ & $<0,001^{c}$ \\
\hline 7 & 1,$40 ; 3,02$ & $<0,001^{\mathrm{C}}$ & 0,$44 ; 2,22$ & $0,004^{c}$ \\
\hline No. leitos hospitalares & $-0,05 ; 0,16$ & 0,297 & $-0,15 ; 0,09$ & 0,583 \\
\hline$\%$ da população com 15-64 anos & 0,$03 ; 0,12$ & $0,002^{c}$ & $-0,08 ;-0,00$ & $0,004^{c}$ \\
\hline \% da população urbana & 0,$02 ; 0,04$ & $<0,001^{\mathrm{c}}$ & 0,$01 ; 0,03$ & $0,003^{c}$ \\
\hline Índice de Gini & 0,$01 ; 0,07$ & $0,012^{c}$ & 0,$00 ; 0,07$ & $0,036^{c}$ \\
\hline No. de testes realizados/ milhão de habitantes & $-0,00 ; 0,00$ & 0,889 & $-0,00 ; 0,00$ & 0,210 \\
\hline & \multicolumn{2}{|c|}{$\begin{array}{l}\text { R-quadrado/R-quadrado } \\
\text { ajustadod: 0,760/0,740 }\end{array}$} & \multicolumn{2}{|c|}{$\begin{array}{l}\text { R-quadrado/R-quadrado } \\
\text { ajustadod: 0,711/0,687 }\end{array}$} \\
\hline
\end{tabular}

determinantes sociais é crucial para que novas sindemias não voltem a ocorrer.

Em conclusão, o estudo evidenciou que a morbimortalidade da COVID-19 esteve correlacionada à carga de condições crônicas, ao envelhecimento da população e à baixa capacidade dos serviços de saúde em realizar testagem e oferecer leitos hospitalares. Isso foi agravado em países ou regiões com elevada desigualdade social, caracterizando, portanto, uma situação de sindemia.

Contribuição dos autores. TPB participou na concepção da pesquisa, coleta de dados, análise e interpretação dos achados, redação e revisão final do manuscrito; FBPC participou na concepção da pesquisa, coleta de dados, interpretação dos achados e revisão final do manuscrito; ACVR, TZB, LHA e YMA participaram na interpretação dos achados e revisão final do manuscrito; FLS participou na revisão final do manuscrito; RAA participou na concepção da pesquisa, interpretação dos achados e revisão final do manuscrito. Além disso, destacamos que todos os autores leram e aprovaram a presente versão do manuscrito.

Conflitos de interesse. Nada declarado pelos autores.

Declaração. As opiniões expressas no manuscrito são de responsabilidade exclusiva dos autores e não refletem necessariamente a opinião ou política da RPSP/PAJPH ou da Organização Pan-Americana da Saúde (OPAS).

\section{REFERÊNCIAS}

1. World Health Organization (WHO). Coronavirus disease 2019 (COVID-19): situation report, 67. Genebra: WHO; 2020. Disponível em: https:/ / apps.who.int/iris/handle/10665/331613 Acessado em 11 de outubro de 2021.

2. Pinho SB. A Atenção primária à saúde no contexto da COVID-19. HU Rev. 2020;46:1-2.

3. Wildman J. COVID-19 and income inequality in OECD countries. Eur J Health Econ. 2021;22(3):455-62.

4. Horton R. COVID-19 is not a pandemic. Lancet. 2020;395(10228): 935.

5. Mendenhall E. The COVID-19 syndemic is not global: context matters. Lancet. 2020;396(10264):1731.

6. Rothman K, Greenland S, Lash T. Epidemiologia moderna. $3^{a}$ edição. Porto Alegre: Artmed Editora; 2016.

7. European Centre for Disease Prevention and Control (ECDC). Data on the geographic distribution of COVID-19 cases worldwide as of 14 December 2020. Solna: ECDC; 2020. Disponível em: http:////www.ecdc.europa.eu/en/publications-data/download -todays-data-geographic-distribution-covid-19-cases-worldwide Acessado em 11 de outubro de 2021.

8. World Bank Open Data. Indicators. Washington: World Bank Open Group; 2020. Disponível em: https:/ / data.worldbank.org/indicator Acessado em 11 de outubro de 2021.

9. Worldometer. Worldometers COVID-19 Data. 2021. Disponível em: https://www.worldometers.info/coronavirus/ Acessado em 11 de outubro de 2021.

10. Cleveland WS, Grosse E. Computational methods for local regression. Stat Comput. 1991;1:47-62.

11. Tierney N, Cook D, McBain M, Fay C. naniar: data structures, summaries, and visualisations for missing data. 2021. Disponível em: https:/ / cran.r-project.org/package=naniar Acessado em 11 de outubro de 2021.

12. Box GEP, Cox DR. An analysis of transformations. J R Stat Soc Series B Stat Methodol. 1964;26(2):211-43.

13. Son H, Hyun C, Phan D, Hwang HJ. Data analytic approach for bankruptcy prediction. Expert Syst Appl. 2019;138: 112816. 
14. Venables WN, Ripley BD. Modern applied statistics with S. Nova Iorque: Springer; 2002.

15. Wickhan H. ggplot2: create elegant data visualisations using the grammar of graphics. Disponível em: https://cran.r-project.org/ web/packages/ggplot2/index.html Acessado em 11 de outubro de 2021.

16. Breusch TS, Pagan AR. The Lagrange Multiplier Test and its applications to model specification in econometrics. Rev Econ Stud. 1980;47(1):239-53.

17. Halunga AG, Orme CD, Yamagata T. A heteroskedasticity robust Breusch-Pagan test for Contemporaneous correlation in dynamic panel data models. J Econom. 2017;198(2):209-30.

18. Hyndman RJ, Athanasopoulos G. Forecasting: principles and practice. $2^{a}$ edição. Austin: OTexts; 2018.

19. Chein F. Introdução aos modelos de regressão linear: um passo inicial para compreensão da econometria como uma ferramenta de avaliação de políticas públicas. 2019. Disponível em: https:// repositorio.enap.gov.br/bitstream/1/4788/1/Livro_Regress $\%$ C3\%A3o\%20Linear.pdf Acessado em 11 de outubro de 2021.

20. Lee VJ, Ho M, Kai CW, Aguilera X, Heymann D, Wilder-Smith A. Epidemic preparedness in urban settings: new challenges and opportunities. Lancet Infect Dis. 2020;20(5);527-9.

21. Liu L. Emerging study on the transmission of the Novel Coronavirus (COVID-19) from urban perspective: Evidence from China. Cities. 2020;103:102759.
22. Chen T, Wu D, Chen H, Yan W, Yang D, Chen G, et al. Clinical characteristics of 113 deceased patients with coronavirus disease 2019: retrospective study. BMJ. 2020;368:m1091.

23. United Nations Development Programme (UNDP). Regional human development report 2021. Nova Iorque: UNDP; 2021. Disponível em: https://www.latinamerica.undp.org/content/rblac/ en/home/library/regional-human-development-report-2021.html Acessado em 11 de outubro de 2021.

24. Solis J, Franco-Paredes C, Henao-Martínez AF, Krsak M, Zimmer $\mathrm{SM}$. Structural vulnerability in the US revealed in three waves of COVID-19. Am J Trop Med Hyg. 2020;103(1):25-7.

25. Redefining vulnerability in the era of COVID-19. Lancet. 2020;395(10230):1089.

26. World Bank Open Data. Cause of death, by non-communicable diseases (\% of total). Washington: World Bank Open Group; 202. Disponível em: https://data.worldbank.org/indicator/SH.DTH. NCOM.ZS Acessado em 11 de outubro de 2021.

Manuscrito recebido em 7 de junho de 2021. Aceito em versão revisada em 19 de outubro de 2021.

\section{COVID-19 morbidity and mortality associated with chronic disorders, healthcare services, and inequity: evidence for a syndemic}

ABSTRACT Objective. To identify factors correlated with the incidence and mortality from COVID-19 and investigate syndemic situations at the global level.

Method. An ecologic study of confirmed COVID-19 cases and deaths was performed using information collected from the European Center for Disease Prevention and Control in 2019 and 2020. World Bank indicators and information obtained from Worldometer Coronavirus were used to characterize the countries. Descriptive analyses and correlations between independent variables were performed, followed by multiple linear regression analysis to identify factors correlated with COVID-19 incidence and mortality.

Results. Data were obtained for 185 countries. Mean case incidence was 16 482/1,000 population, whereas mean COVID-19 mortality was 291/1,000 population, with the highest and lowest rates recorded in North America and East Asia and Pacific respectively. A positive correlation was identified between incidence rate and percent population aged 15 to 64 years, urban population, inequality measured by the Gini coefficient, and six out of the seven regions analyzed (except East Asia and Pacific). Mortality rate was negatively correlated with population aged 0 to 14 years and positively correlated with urban population, inequality measured by the Gini coefficient, and all regions analyzed except East Asia and Pacific.

Conclusions. COVID-19 morbidity and mortality were correlated with the burden of chronic diseases, aging population, and low capacity of healthcare services for testing and providing hospital beds, a scenario complicated by social inequality in countries and regions, indicating a syndemic effect. 


\section{Morbimortalidad por COVID-19 asociada a los problemas crónicos de salud, los servicios de salud y las inequidades: evidencia de sindemia}

RESUMEN Objetivo. Identificar los factores correlacionados con la incidencia de COVID-19 y la mortalidad por esa causa y verificar las situaciones de sindemia a escala mundial.

Métodos. Se realizó un estudio ecológico de casos de COVID-19 y de defunciones confirmadas por esa causa a partir de la información obtenida del Centro Europeo para la Prevención y el Control de las Enfermedades en el 2019 y el 2020. Para caracterizar a los países, se utilizaron indicadores del Banco Mundial y del sitio web de referencia Worldometer Coronavirus. Se hicieron análisis descriptivos y de correlación entre las variables independientes para crear posteriormente un modelo de regresión lineal múltiple con el fin de identificar los factores correlacionados con la incidencia de COVID-19 y la mortalidad por esa causa.

Resultados. Se obtuvieron datos de 185 países. La tasa media de incidencia de casos de COVID-19 fue de 16482 por mil habitantes y la tasa media de mortalidad por esa causa fue de 291 por mil habitantes. Las regiones de América del Norte y de Asia oriental y el Pacífico presentaron los mayores y menores índices, respectivamente. Se observó una correlación positiva de la tasa de incidencia con la proporción del grupo de 15 a 64 años de edad, la población urbana, la desigualdad medida por el coeficiente de Gini y seis de las siete regiones analizadas (excepto Asia oriental y el Pacífico). La tasa de mortalidad presentó una correlación negativa con el grupo de 0 a 14 años de edad y positiva con la población urbana, la desigualdad medida por el coeficiente de Gini y todas las regiones analizadas, excepto Asia oriental y el Pacífico.

Conclusiones. La morbimortalidad por COVID-19 guardó una correlación con la carga de problemas crónicos de salud, el envejecimiento de la población y la poca capacidad de realizar pruebas en los servicios de salud y de ofrecer camas de hospital, cuadro agravado en los países o regiones con una elevada tasa de desigualdad social y característico de una situación de sindemia.

Palabras clave COVID-19; atención a la salud (salud pública); sindémico; pandemias; epidemiología. 